\title{
EFFECTS OF MATURITY STATUS ON ANTHROPOMETRIC MEASURES, PHYSICAL FITNESS, AND TRAINING LOAD IN YOUNG BRAZILIAN SOCCER PLAYERS
}

original paper

() Wroclaw University of Health and Sport Sciences

DOI: https://doi.org/10.5114/hm.2021.104184

\section{MARCELO HENRIQUE ALVES FERREIRA DA SILVA ${ }^{1}$, EDER GONÇALVES ${ }^{2}$, RODRIGO AQUINO ${ }^{3}$, JOÃO ROBERTO LIPAROTTI ${ }^{4}$, MÁRIO JOSÉ ALVES ${ }^{5}$, RANIELLE DAMASCENO RIBEIRO ${ }^{6}$, ANTÓNIO J. FIGUEIREDO ${ }^{2}$}

${ }^{1}$ Faculty of Physical Education, State University of Rio Grande do Norte, Mossoró, Brazil

${ }^{2}$ Research Unit for Sport and Physical Activity, Faculty of Sports Sciences and Physical Education, University of Coimbra, Coimbra, Portugal

${ }^{3}$ LabSport, Post-Graduate Program in Physical Education, Center of Physical Education and Sports,

Federal University of Espírito Santo, Vitória, Brazil

${ }^{4}$ Faculty of Physical Education, Federal University of Rio Grande do Norte, Natal, Brazil

${ }^{5}$ Potiguar University, Natal, Rio Grande do Norte, Brazil

${ }^{6}$ Municipal Department of Sport and Leisure of Natal, Rio Grande do Norte, Brazil

\section{ABSTRACT}

Purpose. The study aimed to (1) verify maturity status effects on training performance indicators in young soccer players; (2) compare the anthropometric, physical fitness, and training performance indicators before and after 10-week training; (3) investigate relationships between anthropometric measures, maturity status, and performance indicators.

Methods. The study involved 34 male soccer players (aged $17 \pm 2$ years). Biological maturation was determined by skeletal age, with left hand radiology. Body height, mass, and adiposity were determined. Physical fitness was measured with field tests, including intermittent aerobic fitness, repeated sprint ability, and lower limb power. The participants were monitored daily over 10 weeks (preseason) through rating of perceived exertion.

Results. Average maturity players showed greater training load $(p=0.04)$ and physical training volume $(p=0.04)$ than early maturity players. Mature players presented higher physical training volume than early maturity players $(p=0.04)$. We verified better performances in intermittent aerobic fitness $(p<0.001, d=1.086)$, vertical jump $(p=0.007, d=0.584)$, best sprint time $(p=0.003, \mathrm{~d}=0.661)$, average sprint time $(p=0.002, d=0.676)$, and sum of the sprint times $(p=0.002$, $d=0.675$ ) after the 10 -week preseason. Physical and technical-tactical training volumes were moderately-largely related to best sprint time $(r=-0.48, r=-0.55$, respectively). Technical-tactical training volume was largely related to intermittent aerobic fitness $(r=0.54)$.

Conclusions. Advanced maturity and mature players presented greater training load and physical training volume than early maturity subjects. Players exhibited improved physical fitness after the 10 -week preseason. Technical-tactical training volume showed a large association with the intermittent aerobic fitness test.

Key words: soccer, performance indicators, physical fitness, sports medicine, sports sciences

\section{Introduction}

Anthropometric, physical fitness, and maturity status indicators have previously been employed during the talent identification process, when players move up across age groups [1, 2], and to monitor the training effect in soccer players [3]. In addition, coaches and researchers report concerns related to differences in morphology, physical fitness, and maturity status of young athletes belonging to the same age group [4]. Thus, to ensure a long sports career of young athletes, coaches and practitioners should monitor training loads considering the maturity status to verify the training-related effects on physical fitness, fatigue symp-

Correspondence address: Rodrigo Aquino, Center of Physical Education and Sports, Federal University of Espírito Santo, Av. Fernando Ferrari, 514 - Goiabeiras, Vitória - ES, Zip Code 29075-910, Brazil, e-mail: aquino.rlq@gmail.com

Received: June 25, 2020

Accepted for publication: September 19, 2020

Citation: Silva M, Gonçalves E, Aquino R, Liparotti JR, Alves MJ, Ribeiro RD, Figueiredo AJ. Effects of maturity status on anthropometric measures, physical fitness, and training load in young Brazilian soccer players. Hum Mov. 2022;23(1);28-36; doi: https://doi.org/10.5114/hm.2021.104184. 
toms, and recovery time-course over the season $[5,6]$. Therefore, the analysis of the maturation stage is fundamental during youth soccer development $[7,8]$. In fact, players in the same age group may be at different levels of biological development, which will provide a significant advantage in physical fitness for those who are in the highest stages of maturation $[9,10]$. Furthermore, maturation stage assessment is necessary for the organization and prescription of training content, considering the training load prescribed in accordance with the biological age and individual physiological capacity [11], reducing the risk of injury, and improving performance $[10,12]$.

Internal (e.g., rating of perceived exertion [RPE], visual analogue scale [VAS]) and external (e.g., total distance covered, accelerations/decelerations) load measures are fundamental to elicit peak performance and reduce the risk of injury over the season [13-18]. However, the effects of biological maturity on the training load have not been fully investigated in youth soccer. This information may encourage coaches to adjust training loads considering the maturity status of the players over the competitive season. In addition, few studies have investigated the load distribution over the season in young soccer players with the purpose of identifying the training-related effects on physical fitness $[14,19,20]$.

A recent systematic review showed 2 studies investigating the internal load (RPE, VAS) of Portuguese and English young players, in which the oldest athletes accumulated greater perceived exertion than younger ones $(\mathrm{U} 18>\mathrm{U17}>\mathrm{U16}>\mathrm{U14})$ [21]. In addition, 1-match weekly internal load varied considerably between young soccer teams (ca. 35\%), with the match accounting for $20-23 \%$ of the total internal load [21]. In fact, these studies provided important insights into player monitoring; however, other training-related measures should be better determined during the season (e.g., physical and technical-tactical training volume, the number of matches) to organize and distribute the training loads over the season [5, 22]. Deeper understanding of the training effect on these parameters, as well as their interactions, could be essential to guide coaches and practitioners during schedule planning over the season.

Therefore, the aim of this study was threefold: (1) to verify the effects of maturity status on training performance indicators in young soccer players; (2) to compare anthropometric variables and physical fitness before and after 10 weeks of training (preseason); and (3) to investigate the relationships between anthropometric measures, maturity status, and performance in- dicators. We expected that advanced maturity players would report higher training load and perceive lower intensity during the sessions compared with their counterparts [20]. In addition, we expected morphology and physical fitness to improve after the 10 weeks of the preseason [23, 24]. Finally, we awaited moderate to large associations between maturity status and performance indicators $[16,25]$.

\section{Material and methods}

\section{Participants}

A total of 34 male young soccer players (age: $17 \pm 2$ years; height: $173 \pm 8 \mathrm{~cm}$; body mass: $64 \pm 13 \mathrm{~kg}$ ) belonging to a professional club of the Northeast region of Brazil participated in this study. The inclusion criteria were: (1) being a soccer player aged 15-18 years; (2) training routine of 5-6 times a week. The goalkeepers were excluded, as well as any outfield players who presented injuries and/or health problems (according to the medical report) that prevented them from participation in training for a period of 7 days.

The player's chronological age (CA) was defined by using the decimal age, calculated with the date of the anthropometric measurements (AD) and the date of birth (BD) of the players [26]:

$$
\mathrm{CA}=(\mathrm{AD}-\mathrm{BD}) / 365
$$

\section{Procedures}

The present study was conducted in nonexperimental conditions in which the research problem was embedded [27]. The players were investigated during the 10 weeks of the preseason. Before beginning the training sessions, all participants were in an inactive period (ca. 40 days - transition phase between the seasons).

Multidimensional variables were considered: the anthropometric profile, maturity status, physical fitness, and training performance indicators. The evaluations were performed at the club's professional soccer training centre (at the measurement and evaluation laboratory and on the natural grass field). The initial evaluations were carried out in the first week of the soccer players' training; they were repeated in the final week of the preparation period. Training load indicators (volume and intensity variables) were collected daily in all training sessions, throughout the preparation period. 
In the first week, before the start of the training session, the athletes underwent assessment of body mass, height, and subcutaneous skinfolds in the measurement and evaluation laboratory. Next, after a 3-minute warm-up, the participants were submitted to a vertical impulse test and then to a horizontal impulse test. Subsequently, already on the training field, the soccer players were divided into groups of 5-6 individuals, to perform an intermittent performance test. A total of 24 hours after the intermittent test, the athletes completed an anaerobic power test. This evaluation routine was repeated at the end of the preparation period.

All evaluations were carried out at the same time of day, from 8 a.m. to 10 a.m. The ambient temperature recorded in the field during the tests ranged between $28^{\circ}$ and $31^{\circ}$, on average, in accordance with the Agricultural Research Corporation of Rio Grande do Norte (www.emparn.rn.gov.br). In the laboratory, a temperature of $24^{\circ}$ was recorded.

The month prior to the start of data collection was dedicated to the training of 3 evaluators (teachers graduated in Physical Education in the state of Rio Grande do Norte). The evaluators' work consisted of organizing tasks for the evaluated groups, arranging the equipment necessary to collect and record data, and measuring and testing aerobic and anaerobic performance. In all cases, the research coordinator was present at the evaluation sites. The evaluators' training ended with the determination of the technical error of measurement and reliability coefficient, through the evaluation of approximately 15 soccer players in one week. Reliability was established with an analysis of replicates of the measurements obtained in a short period of time, expressed as a function of the proportion between the error variance and the inter-individual variance [28]. The reliability coefficients were estimated with the following formula:

$$
\mathrm{R}=1-\left(\mathrm{r}^{2} / \mathrm{s}^{2}\right)
$$

The technical error of measurement was determined by using the formula proposed by Malina et al. [29]:

$$
\mathrm{S}_{\mathrm{e}}=\left(\Sigma \mathrm{z}^{2} / 2 \mathrm{~N}\right)^{0.5}
$$

Regarding the determination of skeletal age, all radiographs were evaluated by the same examiner, and inter-observer reliability was indicated in previous studies [30].

\section{Anthropometric profile}

Player height was assessed with a portable stadiometer with an accuracy of $1 \mathrm{~mm}$ (Sanny ${ }^{\circledR}$, Brazil). Body mass was obtained with electronic scales with a $0.1 \mathrm{~kg}$ precision (Welmy ${ }^{\circledast}$, Brazil), and skinfolds (tricipital, subscapular, suprailiac, thigh, geminal, and abdominal) with a scientific adipometer with an accuracy of $1 \mathrm{~mm}$ (Cescorf ${ }^{\circledast}$, Brazil). All the procedures followed the protocols by Lohman et al. [31].

\section{Physical fitness}

The total distance covered during the Brazilian Soccer Test (BST) was determined to assess the intermittent endurance fitness [32]. For this, a 15-m long and 1.5-m wide course was built at the test site, marked by cones. The test consists of 4 runs of $15 \mathrm{~m}(60 \mathrm{~m})$, with a passive recovery interval of 10 seconds. The aim of the test is for the subject to perform as many repetitions as possible. The speed of the test is controlled by means of sound signals (beeps) recorded on a CD, so that the individual reaches the mark determined exactly at the time of the sound signal. For the sound reproduction in this protocol, a Sony VAIO computer was used and connected to an amplifier box (Mondial MC0-02 Mult conect power bivolt 40W). For every $240 \mathrm{~m}(16 \times 15 \mathrm{~m})$ of distance covered, one stage is completed, with an increase of $1 \mathrm{~km} / \mathrm{h}$ in running speed in the next stage. The test starts (stage 1) at a speed of $9 \mathrm{~km} / \mathrm{h}$ and ends (stage 11) at $19 \mathrm{~km} / \mathrm{h}$, which corresponds to the maximum distance covered of $2880 \mathrm{~m}$. Prior to the test, there is a period ( 3 minutes and $30 \mathrm{sec}-$ onds) of adaptation and warm-up performed at $8 \mathrm{~km} / \mathrm{h}$. The test ends when the subject, for the second consecutive time, is unable to reach the line in time, in accordance with the speed established by the audible signal (objective evaluation), or the subject feels unable (gives up) to continue at the established speed (subjective evaluation). Thus, the total distance covered by the subject is recorded, including the distance covered during the warm-up.

The anaerobic power was assessed with the Running Anaerobic Sprint Test [14]. After a 10-minute warm-up, the test was started. The test consists of 6 sprints at a distance of $35 \mathrm{~m}$. At the end of each run, there is a 10-second recovery interval when the practitioner runs slowly to the starting location for the next sprint. The time obtained in each repetition was recorded by a stopwatch attached to 2 pairs of photoelectric cells (Multisprint - Hidrofit, Belo Horizonte, Brazil). The use of this protocol allows the following 
information to be recorded: best sprint of the first 2 attempts (also used as a speed measure), the worst sprint of the last 2 attempts, mean time spent on the 6 sprints, and the fatigue index (subtraction of the worst sprint from the best sprint divided by the sum of 6). The test was required to respect the following conditions: the start of any of the sprints must be performed in a static way; the runner's recovery rate cannot exceed 10 seconds between the end of the sprint course and the start of the next sprint; at the end of each sprint, the runner is required to maintain the same direction during a space of $10 \mathrm{~m}$ that serves to decelerate; the timekeeper who is recording the runner's recovery time should inform them, at 5-second intervals, of the time left before the start of the next sprint. This performance test was used recently in a study by de Oliveira Matta et al. [10] among young soccer players.

For the evaluation of lower limb power, the vertical impulse protocol was used (countermovement jump, CMJ), widely reported in the literature [30, 31]. For this, we used a force platform (Jump Test 2.0, Belo Horizonte, Brazil). Before the test, the subject performed a 3-minute warm-up, which included muscle-joint mobility and stability exercises and simulating the motor gesture required in the jump protocol. After the warm-up, the participant stood upright, with their hands on their pelvic girdle, passed through the semi-flexed position, and then jumped at maximum height without removing their hands from their waist. From its beginning to end, the movement is continuous, assuming an eccentric and concentric phase before the aerial trajectory. Each player performed 3 attempts, with a 2-minute interval between them, and the best value was recorded (the greatest height achieved).

In addition, to determine the power of lower limbs, the Standing Long Jump protocol was used. Before starting the test, the subject conducted a 3-minute warm-up, through the performance of muscle-joint mobility and stability exercises and simulation of the motor gesture required in the jump protocol. For this test, the athlete initially stood close behind the starting line, perpendicular to a measuring tape (Vonder) fixed on the playing field, with feet parallel to the width of the shoulders, being able to move the arms freely and flex the knees before the jumps. The subject was instructed to jump as far as possible and land on their feet. Each player performed 3 attempts, with a recovery interval of 2 minutes between the jumps, and the longest distance achieved in centimetres was recorded. The distance reached was measured from the starting line to the landing location, defined by the back of the heel of the foot closest to the starting line.

\section{Maturity status}

Biological maturation was determined by skeletal age, on the basis of the radiological examination of the left hand-wrist, with the procedures of the Fels method [33]. The radiological examination was performed only in the first week of the preparation period, in a medical clinic, in the afternoon. Skeletal maturation was diagnosed by an expert, and the categorization of athletes by maturation status was conducted in accordance with Malina et al. [26] considerations. Thus, the following skeletal maturation stages were defined: delayed (delayed/late maturing) - players with a skeletal age below the chronological age by more than 1 year; normomature (on time/average) - players with a skeletal age within the range of \pm 1 year in relation to the chronological age; advanced (advanced/early mature) - soccer players with a skeletal age above the chronological age by more than 1 year; and mature adult or skeletally mature players. In our sample, no individuals with late maturity were identified.

\section{Training performance indicators}

The training indicators used over the 10 weeks were the training volume (minutes), physical training volume (minutes), technical-tactical training volume (minutes), number of matches, RPE, and training load.

The training volume included the games and all training sessions, whether physical or technical-tactical, during the preparation period (10 weeks). The volume of technical-tactical training counted not only the specific technical and tactical activities implemented by the coach, but also the training games. The volume of physical training comprised all the specific activities included by the team's physical trainer aimed at physical qualities (strength, endurance, speed, etc.).

The training load was determined in accordance with the session RPE, i.e., through the product of the total training session volume by the RPE scores (CR10) (training load $=$ session volume $\times$ RPE) [34]. RPE was recorded ca. 30 minutes after each training session and match during the 10 weeks. The process of familiarizing athletes with the RPE scale was carried out before the start of the evaluations and training, in order to standardize the low and high subjective perception of effort. We used the maximum effort made by the soccer players in the intermittent performance test as a reference of the 'maximum' (value 10), and the athlete's state of rest as a reference of the 'minimum' $(\mathrm{RPE}=0)$, so that this measure reflected the global assessment of training. 


\section{Statistical analysis}

The data distribution and homogeneity of variance were checked and confirmed by using the ShapiroWilk and Levene's tests, respectively. The analysis of covariance (ANCOVA - chronological age as covariable) and Bonferroni's post-hoc test were applied to verify the effects of the maturation stage on training indicators. The effect size was defined with the eta squared value $\left(\eta^{2}\right)$. The magnitude of the effect size followed the classification proposed by Cohen [35]. Student's $t$-test for dependent samples served to verify the effects of the 10-week training on anthropometric and physical fitness data. The effect size was defined by using Cohen's $d$ [35]. The relationships between training performance indicators, anthropometric data, and physical fitness were obtained with the Pearson correlation coefficient $(r)$. Correlations were determined by verifying the association between training load indicators and alterations (mean post-pre change) in morphology and physical fitness performance. The magnitude of correlation coefficients was defined in accordance with Hopkins [36]. All analyses were performed with the SPSS $^{\circledast}$ software, version 20.0 (Chicago, USA). Statistical significance was set at $p<0.05$.

\section{Ethical approval}

The research related to human use has complied with all the relevant national regulations and institutional policies, has followed the tenets of the Declaration of Helsinki, and has been approved by the University of Coimbra, Portugal (Faculty of Sports Sciences and Physical Education) Scientific Committee.

\section{Informed consent}

Informed consent has been obtained from the directors of the club, the players' legal guardians, and the players included in this study.

\section{Results}

Table 1 shows the results of covariance analysis with chronological age as a covariate. The analysed indicators - training load and volume, volume of physical training and technical-tactical training, and number of games - corresponded to the sum of the entire preparation period (10 weeks). RPE refers to the mean value obtained in the 10 weeks. We found large significant effects of maturity status on training load and physical training volume. The average maturity players showed greater training load $(p=0.04)$ and physical training volume ( $p=0.04$ ) than early maturity players. In addition, the mature players presented higher values of physical training volume than early maturity players $(p=0.04)$. The maturity status did not exert significant effects on RPE, training volume, technical-tactical training volume, or the number of matches.

Table 2 presents the effects of 10 weeks of training on anthropometric and physical fitness data. Anthropometric measures did not exhibit significant effects between the pre- and post-training status. However, we observed better performance in BST, CMJ, best sprint time, average sprint time, and sum of the sprint times after the training protocol.

Table 3 illustrates the relationships between anthropometric measures, physical fitness, and training performance indicators. Training volume presented large associations with best sprint time $(r=$ $-0.52, p=0.03)$. In addition, best sprint time exhibited moderate and high associations with physical training volume $(r=-0.48, p=0.04)$ and technicaltactical training volume $(r=-0.55, p=0.02)$, respectively. Finally, there was a large correlation between technical-tactical training volume and BST $(r=0.54$, $p=0.02$ ). No significant correlations were revealed of RPE or training load with anthropometric or functional performance variables.

Table 1. Effects of maturity status on training performance indicators in young soccer players (adjusted mean, standard error, and analysis of covariance with chronological age as covariate)

\begin{tabular}{lccccc}
\hline & \multicolumn{4}{c}{ Maturity status } \\
\cline { 2 - 4 } Training performance indicators & $\begin{array}{c}\text { Average } \\
(n=9)\end{array}$ & $\begin{array}{c}\text { Early } \\
(n=14)\end{array}$ & $\begin{array}{c}\text { Mature } \\
(n=11)\end{array}$ & $F$ & \\
\hline Training load (AU) & $13,786 \pm 745$ & $11,028 \pm 693.5$ & $13,801 \pm 773$ & 4.063 & 0.027 \\
Training volume (min) & $3852 \pm 199$ & $3201 \pm 185.5$ & $3891 \pm 206.5$ & 3.302 & 0.051 \\
Rating of perceived exertion (AU) & $3.31 \pm 0.06$ & $3.21 \pm 0.05$ & $3.29 \pm 0.06$ & 0.634 & 0.537 \\
Physical training volume (min) & $2076 \pm 88$ & $1747.5 \pm 82$ & $2100 \pm 91$ & 4.358 & 0.022 \\
Technical-tactical training volume (min) & $1672 \pm 106$ & $1413 \pm 99$ & $1719.5 \pm 110$ & 2.062 & 0.145 \\
Number of matches & $5.60 \pm 0.56$ & $5.28 \pm 0.53$ & $6.70 \pm 0.59$ & 1.465 & 0.247 \\
\hline AU & & & &
\end{tabular}

$\mathrm{AU}$ - arbitrary units 
Table 2. Effects of 10 weeks of training on anthropometric and physical fitness data in young soccer players (mean \pm standard deviation)

\begin{tabular}{|c|c|c|c|c|c|c|}
\hline \multirow{2}{*}{ Variables } & \multicolumn{2}{|c|}{10 weeks } & \multirow{2}{*}{ MC (\%) } & \multirow{2}{*}{$t$} & \multirow{2}{*}{$p$} & \multirow{2}{*}{$d$} \\
\hline & Before & After & & & & \\
\hline \multicolumn{7}{|l|}{ Anthropometric measures } \\
\hline Height $(\mathrm{cm})$ & $172.9 \pm 5.6$ & $173.7 \pm 5.8$ & $0.7 \pm 1.5$ & 2.569 & 0.016 & - \\
\hline Body mass (kg) & $64.2 \pm 7.6$ & $65.0 \pm 6.8$ & $0.8 \pm 2.6$ & 1.695 & NS & - \\
\hline Adiposity (mm) & $52.4 \pm 12.9$ & $52.4 \pm 10.3$ & $0.0 \pm 6.6$ & 0.000 & NS & - \\
\hline \multicolumn{7}{|l|}{ Physical fitness } \\
\hline BST (m) & $1750 \pm 140$ & $1920 \pm 174$ & $169 \pm 159$ & 5.733 & 0.001 & 1.086 \\
\hline SLJ (cm) & $213.9 \pm 13.4$ & $212.9 \pm 15.4$ & $-1.0 \pm 6.8$ & -0.673 & NS & - \\
\hline $\mathrm{CMJ}(\mathrm{cm})$ & $35.3 \pm 3.4$ & $36.7 \pm 2.9$ & $1.3 \pm 2.3$ & 2.927 & 0.007 & 0.584 \\
\hline Best sprint time (s) & $5.2 \pm 0.3$ & $5.2 \pm 0.2$ & $-0.1 \pm 0.1$ & -3.318 & 0.003 & 0.661 \\
\hline Average sprint time (s) & $5.6 \pm 0.3$ & $5.5 \pm 0.2$ & $-0.1 \pm 0.2$ & -3.477 & 0.002 & 0.676 \\
\hline Sum of the sprint times (s) & $33.8 \pm 1.8$ & $33.1 \pm 1.2$ & $-0.7 \pm 1.1$ & -3.477 & 0.002 & 0.675 \\
\hline Fatigue index (\%) & $7.3 \pm 2.4$ & $6.8 \pm 2.0$ & $-0.5 \pm 2.2$ & -1.211 & NS & - \\
\hline
\end{tabular}

MC - mean change between pre- and post-training tests, NS - not significant

BST - Brazilian Soccer Test, SLJ - standing long jump, CMJ - countermovement jump

Table 3. Partial correlation (considering chronological age) between training performance indicators, anthropometric data, physical fitness, and skeletal age in young soccer players

\begin{tabular}{|c|c|c|c|c|c|}
\hline \multirow[b]{2}{*}{ Variables } & \multicolumn{5}{|c|}{ Training performance indicators } \\
\hline & $\begin{array}{l}\text { Rating of perceived } \\
\text { exertion }\end{array}$ & $\begin{array}{l}\text { Training } \\
\text { volume }\end{array}$ & $\begin{array}{l}\text { Training } \\
\text { load }\end{array}$ & $\begin{array}{l}\text { Physical } \\
\text { training volume }\end{array}$ & $\begin{array}{l}\text { Technical-tactical } \\
\text { training volume }\end{array}$ \\
\hline $\mathrm{SA} / \mathrm{CA}$ ratio & -0.242 & -0.092 & -0.197 & 0.018 & -0.013 \\
\hline SA (years) & -0.229 & -0.092 & -0.193 & 0.014 & -0.016 \\
\hline Height (cm) & 0.019 & 0.302 & 0.299 & 0.302 & 0.232 \\
\hline Body mass (kg) & -0.117 & 0.040 & -0.021 & 0.154 & -0.116 \\
\hline Adiposity (mm) & -0.058 & 0.023 & -0.008 & 0.207 & -0.153 \\
\hline $\operatorname{BST}(\mathrm{m})$ & -0.352 & 0.425 & 0.183 & 0.370 & $0.54^{*}$ \\
\hline SLJ (cm) & 0.026 & 0.073 & 0.123 & 0.079 & 0.078 \\
\hline $\mathrm{CMJ}(\mathrm{cm})$ & -0.181 & 0.296 & 0.128 & 0.253 & 0.315 \\
\hline Best sprint time (s) & 0.068 & $-0.52 *$ & -0.409 & $-0.48 *$ & $-0.55^{*}$ \\
\hline Average sprint time (s) & 0.003 & -0.430 & -0.344 & -0.431 & -0.433 \\
\hline Sum of the sprint times (s) & 0.003 & -0.430 & -0.344 & -0.431 & -0.433 \\
\hline Fatigue index (\%) & -0.089 & 0.063 & 0.035 & 0.021 & 0.097 \\
\hline
\end{tabular}

SA - skeletal age, CA - chronological age, BST - Brazilian Soccer Test

SLJ - stand long jump, CMJ - countermovement jump

${ }^{*} p<0.05$

\section{Discussion}

This study investigated the effects of maturity status on training performance indicators in young soccer players. Furthermore, we compared the anthropometric measures, physical fitness, and training performance indicators before and after 10 weeks of training. Additionally, we verified the relationships between anthropometric data, maturity status, and training performance indicators. The main findings were as follows: (1) average maturity players showed greater training load and physical training volume than early maturity players; mature players presented higher values of physical training volume than early maturity players; (2) we demonstrated trainingrelated effects on BST, CMJ, best sprint time, average sprint time, and sum of the sprint times; (3) there were moderate and high correlations of best sprint time with physical and technical-tactical training time, respectively; technical-tactical training volume was also largely related to BST. 
Here, mature and average maturity players demonstrated higher training load and training volume compared with early maturity players. Wrigley et al. [20] identified greater response in the training load among the older category (U18) than in the lower age categories. Additionally, an increased training load was observed in the older age group (U18 > U17 > U16 $>$ U14) [20]. This reflects the combined input from both the coaches and sports science team to adhere to the training principles advocated for long-term youth soccer development. Thus, the training load should be structured in relation to the maturity status of the players in order to achieve athletic development whilst minimizing the risk of injuries [20,37].

In the current study, there was no significant effect of youth maturation stage on the RPE training indicator. In spite of this, it seems that there was a tendency for average maturity and mature players to perceive a greater effort to support the same external load imposed. Brink et al. [14] believe that the dissimilarity related to RPE in the same category is due to the physical differences between the players and the sudden increase in the load in the transition of soccer players from one category to another. Similarly, maturity effects were not identified for the number of matches, contrasting previous findings that coaches preferred to select mature young soccer players [38, 39]. In addition, a superior maturity status and anthropometric and fitness measures were verified in young players (U15, U16) who were successful in attaining international or professional level compared with players who remained amateur [40]. Furthermore, athletes advanced in age and/or maturation, and/ or presenting greater body size exhibited systematically greater physical fitness and match running performances than their younger, less mature, and/or smaller teammates [41]. Therefore, these findings confirm the importance of coaches and practitioners considering the maturation status when assessing young soccer players on the basis of anthropometric measures, physical fitness, and training performance indicators.

Concerning the training effects, we verified superior performance in intermittent endurance fitness, sprint time, repeated sprint ability, and lower limb power after 10 weeks of training (total training load: $12,655 \pm 2614$ arbitrary units [AU]; total training volume: $3596.5 \pm 654$ minutes; mean RPE scores: $3 \pm 0.2$ ). Miranda et al. [42] also showed positive changes in anthropometric measures and physical fitness after 10 weeks of soccer training in a group of players aged 15-18 years (total training load: 15,341
AU; total training volume: 4110 minutes; mean RPE scores: 3). In Brazilian U16 soccer players, 22 weeks of periodization training focused on technical-tactical actions (mean training volume: 110 minutes; mean RPE scores: 6) promoted positive changes in match physical and tactical performance [23]. Recently, Aquino et al. [24] observed that short-term pre-season (6-week) training with an emphasis on small-sided games and strength training contributed to benefits in several physical fitness measures and match performance. These findings corroborate the present study, suggesting that appropriate load monitoring and training content distribution are essential to optimize player physical and technical-tactical adaptation.

The current study found moderate and high associations between the best sprint time and the volume of physical and technical-tactical training, respectively. In addition, technical-tactical training volume was largely associated with intermittent aerobic fitness (e.g., distance covered during BST). In contrast, training load and RPE scores were not correlated with indicators of physical fitness or anthropometric data. In Spanish U18 players, training volume (mean: 360 minutes) and training load (mean: $1500 \mathrm{AU}$ ) were largely related to changes in aerobic fitness [25]. In addition, Brink et al. [19] showed that weekly training volume presented a large association with intermittent aerobic fitness in Dutch elite U19 players. Gómez-Díaz et al. [16] identified a large association between perceived exertion over the week (mean RPE scores: 4) and high-speed (> $19.8 \mathrm{~km} / \mathrm{h}$ ) distance covered during matches.

This study has some limitations that should be acknowledged. We did not monitor the players throughout the full season, and the consistency of the reported relationships and training effects may change over the competitive period. Moreover, the training load was obtained by using a subjective internal measure (i.e., RPE scores). Some confounding variables, such as temperature and humidity, were not controlled in all training sessions.

Additional studies should be carried out throughout the season and in younger training categories, verifying the effects of skeletal maturation stages on these and other training indicators (external and internal load), such as technical and tactical aspects (e.g., decision-making). We also suggest studies that use regression analysis to understand which physical parameters can best predict training load.

The analysis of the maturation, performance, and training dimensions in different training categories has decisive practical implications in the prescription and 
evaluation of the training effect. The variables analysed in the current study may contribute to the systematization, monitoring, and transition of young soccer players throughout the training process. Finally, the results reported in this article may contribute to training planning, as well as to developing scientific knowledge in the area of sports training.

\section{Conclusions}

In summary, the advanced maturity and mature young players presented greater training load and physical training volume compared with early maturity players. In addition, the athletes exhibited improved physical fitness after the 10 weeks of preseason (i.e., intermittent endurance fitness, sprint time, repeated sprint ability, and vertical lower limb power). A moderate to large association was observed between the best time during the sprint test and physical and technical-tactical training volume. Finally, the technical-tactical training volume showed a large association with the intermittent aerobic fitness test.

\section{Acknowledgments}

The authors would like to thank the coaching staff and players who participated in this study.

\section{Disclosure statement}

No author has any financial interest or received any financial benefit from this research.

\section{Conflict of interest}

The authors state no conflict of interest.

\section{References}

1. Reilly T, Bangsbo J, Franks A. Anthropometric and physiological predispositions for elite soccer. J Sports Sci. 2000;18(9):669-683; doi: 10.1080/02640410050 120050.

2. Carling C, Le Gall F, Malina RM. Body size, skeletal maturity, and functional characteristics of elite academy soccer players on entry between 1992 and 2003. J Sports Sci. 2012;30(15):1683-1693; doi: 10.1080/0264 0414.2011.637950.

3. Buchheit M, Simpson BM, Peltola E, Mendez-Villanueva A. Assessing maximal sprinting speed in highly trained young soccer players. Int J Sports Physiol Perform. 2012;7(1):76-78; doi: 10.1123/ijspp.7.1.76.

4. Malina RM, Cumming SP, Rogol AD, Coelho e Silva MJ, Figueiredo AJ, Konarski JM, et al. Bio-banding in youth sports: background, concept, and application. Sports Med. 2019;49(11):1671-1685; doi: 10.1007/s40279019-01166-x.
5. Bourdon PC, Cardinale M, Murray A, Gastin P, Kellmann M, Varley MC, et al. Monitoring athlete training loads: consensus statement. Int J Sports Physiol Perform. 2017;12(Suppl. 2):S2161-S2170; doi: 10.1123/ IJSPP.2017-0208.

6. Kokstejn J, Musalek M, Wolanski P, Murawska-Cialowicz E, Stastny P. Fundamental motor skills mediate the relationship between physical fitness and soccerspecific motor skills in young soccer players. Front Physiol. 2019;10:596; doi: 10.3389/fphys.2019.00596.

7. Figueiredo AJ, Gonçalves CE, Coelho e Silva MJ, Malina RM. Characteristics of youth soccer players who drop out, persist or move up. J Sports Sci. 2009;27(9):883891; doi: 10.1080/02640410902946469.

8. Philippaerts RM, Vaeyens R, Janssens M, Van Renterghem B, Matthys D, Craen R, et al. The relationship between peak height velocity and physical performance in youth soccer players. J Sports Sci. 2006;24(3):221230; doi: 10.1080/02640410500189371.

9. Figueiredo AJ, de Oliveira Matta M, Werneck FZ. Morphological and functional issues in the development of young male athletes. Kinesiol Slov. 2014;20(3):27-35.

10. De Oliveira Matta M, Figueiredo AJ, Garcia ES, Wernek FZ, Seabra A. Relative age effect on anthropometry, biological maturation and performance of young soccer players. Rev Bras Cineantropom Desempenho Hum. 2015;17(3):257-268; doi: 10.5007/1980-0037.2015v1 $7 \mathrm{n} 3 \mathrm{p} 257$.

11. Gabbett TJ, Whyte DG, Hartwig TB, Wescombe H, Naughton GA. The relationship between workloads, physical performance, injury and illness in adolescent male football players. Sports Med. 2014;44(7):989-1003; doi: 10.1007/s40279-014-0179-5.

12. Kunrath CA, Gonçalves E, Teoldo I, Barbosa MAM. Maturation somatic and physical fitness in young soccer players [in Portuguese]. Rev Andal Med Deporte. 2017;10(4):187-191; doi: 10.1016/j.ramd.2016.01.002.

13. Foster C, Daines E, Hector L, Snyder AC, Welsh R. Athletic performance in relation to training load. Wis Med J. 1996;95(6):370-374.

14. Brink MS, Frencken WGP, Jordet G, Lemmink KAPM. Coaches' and players' perceptions of training dose: not a perfect match. Int J Sports Physiol Perform. 2014; 9(3):497-502; doi: 10.1123/ijspp.2013-0009.

15. Brink MS, Visscher C, Arends S, Zwerver J, Post WJ, Lemmink KA. Monitoring stress and recovery: new insights for the prevention of injuries and illnesses in elite youth soccer players. Br J Sports Med. 2010;44(11): 809-815; doi: 10.1136/bjsm.2009.069476.

16. Gómez-Díaz AJ, Pallarés JG, Díaz A, Bradley PS. Quantification of physical and psychological loads: differences in competitive levels and the effects on match results [in Spanish]. Rev Psicol Deporte. 2013;22(2):463-469.

17. Alexandre D, da Silva CD, Hill-Haas S, Wong DP, Natali AJ, De Lima JRP, et al. Heart rate monitoring in soccer: interest and limits during competitive match play and training, practical application. J Strength Cond 
Res. 2012;26(10):2890-2906; doi: 10.1519/JSC.0b013 e3182429ac7.

18. Clemente FM, Rabbani A, Ferreira R, Araújo JP. Drops in physical performance during intermittent small-sided and conditioned games in professional soccer players. Hum Mov. 2020;21(1):7-14; doi: 10.5114/hm.2020. 88148.

19. Brink MS, Nederhof E, Visscher C, Schmikli SL, Lemmink KAPM. Monitoring load, recovery, and performance in young elite soccer players. J Strength Cond Res. 2010;24(3):597-603; doi: 10.1519/JSC.0b013e31 81c4d38b.

20. Wrigley R, Drust B, Stratton G, Scott M, Gregson W. Quantification of the typical weekly in-season training load in elite junior soccer players. J Sports Sci. 2012; 30(15):1573-1580; doi: 10.1080/02640414.2012.709265.

21. Rico-González M, Mendez-Villanueva A, Los Arcos A. Training load periodization in soccer with one official match a week: a systematic review. In: Alvira DC, RayaGonzález J (eds.), An essential guide to sports performance. New York: Nova Science Publishers; 2020; 123-166.

22. Clemente FM, Sarmento H. The effects of small-sided soccer games on technical actions and skills: a systematic review. Hum Mov. 2020;21(3):100-119; doi: 10.5114/hm.2020.93014.

23. Aquino RLQT, Cruz Gonçalves LG, Palucci Vieira LH, Oliveira LP, Alves GF, Pereira Santiago PR, et al. Periodization training focused on technical-tactical ability in young soccer players positively affects biochemical markers and game performance. J Strength Cond Res. 2016;30(10):2723-2732; doi: 10.1519/JSC.00000000 00001381.

24. Aquino R, Cruz Gonçalves LG, Bagatin R, Petiot GH, Puggina EF. Short-term training based on small-sided games improved physical and match performance in young football players. Sci Sports. 2020;35(3):180-184; doi: 10.1016/j.scispo.2019.01.006.

25. Gil-Rey E, Lezaun A, Los Arcos A. Quantification of the perceived training load and its relationship with changes in physical fitness performance in junior soccer players. J Sports Sci. 2015;33(20):2125-2132; doi: 10.1080/ 02640414.2015.1069385.

26. Malina RM, Peña Reyes ME, Figueiredo AJ, Coelho e Silva MJ, Horta L, Miller R, et al. Skeletal age in youth soccer players: implication for age verification. Clin J Sport Med. 2010;20(6):469-474; doi: 10.1097/JSM.0b 013e3181f827ea.

27. Cruz Gonçalves LG, Kalva-Filho CA, Nakamura FY, Rago V, Afonso J, de Souza Bedo BL, et al. Effects of match-related contextual factors on weekly load responses in professional Brazilian soccer players. Int J Environ Res Public Health. 2020;17(14):5163; doi: 10.3390/ ijerph17145163.

28. Mueller WH, Martorell R. Reliability and accuracy of measurement. In: Lohman TG, Roche AF, Martorell R (eds.), Anthropometric standardization reference manual. Champaign: Human Kinetics; 1988; 83-86.
29. Malina RM, Hamill PVV, Lemeshow S. Selected body measurements of children 6-11 years. Vital Health Stat. 1973;123:1-48.

30. Figueiredo AJ, Gonçalves CE, Coelho e Silva MJ, Malina RM. Youth soccer players, 11-14 years: maturity, size, function, skill and goal orientation. Ann Hum Biol. 2009;36(1):60-73; doi: 10.1080/03014460802570584.

31. Lohman TG, Roche AF, Martorell R (eds.). Anthropometric standardization reference manual. Champaign: Human Kinetics; 1988.

32. Silva MHAF, Liparotti JR, Figueiredo AJ. The application of a specific performance test for young footballers [in Portuguese]. Ann Res Sport Phys Act. 2015:6: 25-41; doi: 10.14195/2182-7087_6_2.

33. Roche AF, Chumlea WC, Thissen D. Assessing the skeletal maturity of the hand-wrist: Fels method. Springfield: Charles C. Thomas; 1988.

34. Foster C, Florhaug JA, Franklin J, Gottschall L, Hrovatin $\mathrm{LA}$, Parker S, et al. A new approach to monitoring exercise training. J Strength Cond Res. 2001;15(1): 109-115; doi: 10.1519/00124278-200102000-00019.

35. Cohen J. Statistical power analysis for the behavioral sciences. New York: Lawrence Erlbaum Associates; 1988.

36. Hopkins WG. Measures of reliability in sports medicine and science. Sports Med. 2000;30(1):1-15; doi: 10.2165/ 00007256-200030010-00001.

37. Naughton G, Farpour-Lambert NJ, Carlson J, Bradney M, Van Praagh E. Physiological issues surrounding the performance of adolescent athletes. Sports Med. 2000;30(5):309-325; doi: 10.2165/00007256-20003 0050-00001.

38. Aquino R, Alves IS, Padilha MB, Casanova F, Puggina EF, Maia J. Multivariate profiles of selected versus nonselected elite youth Brazilian soccer players. J Hum Kinet. 2017;60:113-121; doi: 10.1515/hukin-2017-0094.

39. Vaeyens R, Malina RM, Janssens M, Van Renterghem B, Bourgois J, Vrijens J, et al. A multidisciplinary selection model for youth soccer: the Ghent Youth Soccer Project. Br J Sports Med. 2006;40(11):928-934; doi: 10.1136/ bjsm.2006.029652.

40. Le Gall F, Carling C, Williams M, Reilly T. Anthropometric and fitness characteristics of international, professional and amateur male graduate soccer players from an elite youth academy. J Sci Med Sport. 2010;13(1): 90-95; doi: 10.1016/j.jsams.2008.07.004.

41. Buchheit M, Mendez-Villanueva A. Effects of age, maturity and body dimensions on match running performance in highly trained under-15 soccer players. J Sports Sci. 2014;32(13):1271-1278; doi: 10.1080/02640 414.2014.884721.

42. Miranda REEPC, Antunes HKM, Pauli JR, Puggina EF, da Silva ASR. Effects of 10-week soccer training program on anthropometric, psychological, technical skills and specific performance parameters in youth soccer players. Sci Sports. 2013;28(2):81-87; doi: 10.1016/j.scispo. 2012.02.005. 